\title{
Anatolii D. Panferov, Alexey V. Makhankov, Alexander V. Trunov The use of an adaptive mesh based on a quadtree for modeling the final state of a quantum field system under pulsed external action
}

\begin{abstract}
.
The success of using mathematical models that determine the behavior of quantum field systems in parametric spaces critically depends on the level of optimization of the procedure of finding the solution. The paper considers the problem of calculating the density of carriers arising in graphene as a result of the action of a pulsed electric field. The basis of the model is a system of kinetic equations that provide the calculation of the residual distribution function. Its integration over momentum space gives the desired carrier density.

The problem lies in the high computational complexity of covering the momentum space with a uniform mesh, which provides an accurate calculation of the density for various parameters of the field momentum. Moreover, the model does not contain criteria for determining satisfactory mesh parameters. The article proposes and implements a procedure for constructing an adaptive mesh in the form of a quadtree having a variable size of covering squares. The procedure is iterative and combined with the process of calculating the values of the distribution function.
\end{abstract}

Key words and phrases: numerical simulation, adaptive mesh, quadtree, graphene.

2010 Mathematics Subject Classification: 81T80; 81T40, 82C20

\section{Introduction}

Modeling the response processes of graphene to the action of an external electric field makes it possible to evaluate the characteristics of promising solutions based on this material for a wide range of frequencies, intensities, and pulse durations [1-4]. The inclusion of an external field starts the process of the creation of free carriers and their subsequent evolution. Their momentum determines the properties of carriers. Due to the two-dimensionality of the system under consideration, the momentum

Supported by RFBR according to the research project 18-07-00778.

(c) A. D. Panferov, A. V. Makhankov, A. V. Trunov, 2020

(C) Saratov State University, 2020

(c) Program Systems: Theory and Applications (design), 2020 
space (the space of wave vectors) is also two-dimensional. In the simplest case, we can assume that before the external field is turned on, this space is empty, and the states in it begin to populate as a result of the electric field. Since, in the case under consideration, the carriers obey Fermi statistics, any of the states can be occupied by only one quasiparticle. The process of the population of states is probabilistic by nature, and it is convenient to describe it by the distribution function $0 \leq f\left(p_{1}, p_{2}, t\right) \leq 1$, which represents the probability of detecting the state $\left\{p_{1}, p_{2}\right\}$ populated at time $t$. The process model used determines the procedure for calculating $f\left(p_{1}, p_{2}, t\right)$ for the selected point of the momentum space $-\pi \leq p_{1} \leq \pi,-\pi \leq p_{2} \leq \pi$ (first Brillouin zone) [5-7]. The procedure implements a numerical solution of the quantum kinetic equation formulated as a system of ordinary differential equations. It is universal for any point in the momentum space and parameters of the external field.1

The observed characteristics of the simulated process are determined by averaging over the calculated $f\left(p_{1}, p_{2}, t\right)$. For example, to determine the surface density of carriers, it is necessary to calculate an integral of the form: $n(t)=g \int f\left(p_{1}, p_{2}, t\right) d p_{1} d p_{2}$, where $g$ is a constant normalization coefficient. To calculate integrals of this kind, we need a fairly detailed knowledge of the behavior of the distribution function in the entire domain. The regular meshes with a constant step do not provide an optimal solution in this case [8]. The article demonstrates the capabilities of the procedure for constructing a regular mesh with a variable step, chosen taking into account the nature of the behavior of the distribution function. We consider only the final state of the distribution function when, after switching off the external field, the evolution in the system became frozen, and the distribution function ceases to depend on time.

\section{Carrier distribution function behavior in momentum space}

The space of states considered in this formalism, although limited by the first Brillouin zone, is very large from a physical point of view. Its complete occupation would ensure the carrier density at the level of $n_{\max }=1.32 \times 10^{16} 1 / \mathrm{cm}^{2}$ which is unattainable for many reasons (primarily, due to the presence of dissipative processes). The values obtained by modeling realistic situations are several orders of magnitude less than $n \ll n_{\max }$. In general, it does not follow from this that $\left(\forall\left\{p_{1}, p_{2}\right\}\right)$ $\left(f\left(p_{1}, p_{2}\right) \ll 1\right)$, i.e. the distribution function can take on sufficiently large values of $f\left(p_{1}, p_{2}\right) \approx 1$ in some areas of the state space. If so, then in most of the state space $f\left(p_{1}, p_{2}\right) \approx 0$ and they do not contribute to the integral characteristics. 


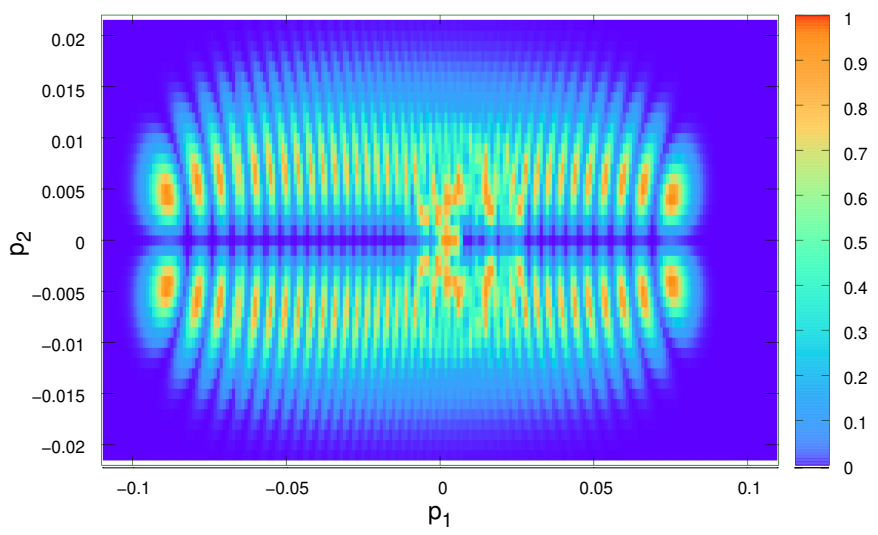

FIgURE 1. Distribution function $f\left(p_{1}, p_{2}\right)$ after the action of the pulse field with parameters: duration $2 \times 10^{-12} \mathrm{~s}$, carrier frequency $2 \times 10^{12} \mathrm{~Hz}$, amplitude $3 \times 10^{4} \mathrm{~V} / \mathrm{cm}$

As an example, consider the procedure for simulating the action of a short electric field pulse with parameters corresponding to those used in [2]. Figure 1 shows the results of calculating the residual distribution function formed during the pulse. 1

The figure is built on a regular mesh with a constant step of $222 \times 46$ for the region $-0.1105 \leq p_{1} \leq 0.1105,-0.0225 \leq p_{2} \leq 0.0225$ [9]. All non-zero values of the distribution function are localized in this region, although it occupies only $2.519 \times 10^{-4}$ part of the first Brillouin zone. The figure shows a very complex behavior of the distribution function with a large number of local peaks with values of $f\left(p_{1}, p_{2}\right) \approx 1$, which are adjacent to the regions of small values of $f\left(p_{1}, p_{2}\right) \approx 0$.

More detailed information on the features of the forms of the distribution function is collected in [7]. Based on the data presented there, we can conclude that when changing the parameters of the external acting field (amplitude, duration, nature of the dependence on time), both the localization region and the form of the dependence $f\left(p_{1}, p_{2}\right)$ vary within a vast range. In each case, it is necessary to solve two partially independent tasks to determine them. The first is to remove from consideration areas with near-zero values that do not make a significant contribution to the observed values with minimal resources. The second is to restore $f\left(p_{1}, p_{2}\right)$ in the localization region with the given accuracy.

When using regular meshes with a constant step, specialists usually 
implement this procedure by a sequence of meshes of various sizes and densities with an analysis of the results of each iteration. To automate this procedure, we propose a mechanism for constructing an adaptive regular mesh with a variable step.

\section{Adaptive mesh algorithm}

Step bisection is usual when solving the problem of optimizing the numerical integration procedure. For example, when using the Runge rule to estimate the error of quadrature formulas and in adaptive algorithms. This procedure is generalized to two-dimensional and three-dimensional cases by using quad - and octo - trees. For the two-dimensional space $\left(p_{1}, p_{2}\right)$ under consideration, the quadtree allows us to determine its complete coverage by squares whose aspect ratio belongs to a series of values $2^{N}$, where $N=1,2,3 \ldots$ is a series of natural numbers. The value of the function within each such square is taken equal to its value at the central point.

To ensure universality when working with arbitrary parameters of the simulated process, the adaptive mesh construction area should be the entire first Brillouin zone $-\pi \leq p_{1} \leq \pi,-\pi \leq p_{2} \leq \pi$. It acts as the root of the quadtree. Strictly speaking, the value of the distribution function for the root must be estimated at point $p_{1}=0, p_{2}=0$. However, there is a singularity at this point in the method of determining the coefficients of the system of equations used. The problem can be circumvented by introducing a very small offset $\delta \ll 2 \pi$ from the center of the Brillouin zone. In the case of constructing a quadtree with the number of generations $N$, the offset should be dangerously small $\delta \ll 2 \pi / 2^{N}$. In the version of the algorithm adopted for implementation, we abandon this method of circumventing the singularity and simply exclude the root node from consideration, placing it exactly at the point $p_{1}=0, p_{2}=0$. An additional advantage is a guarantee that the nodes of the older generations will never find themselves at this problem point.

We construct the adaptive mesh by phasing up (squaring) its selected elements. Non-split elements are leaves of a quadtree. Elements on which the partitioning procedure was performed are intermediate nodes. The selection criteria for the elements to be further partitioned will play a key role in the success of the algorithm. Each step of the algorithm will translate part of the leaves of the last generation into the status of intermediate nodes, building on them the leaves of the new generation and complete with the calculation of the distribution function values in the fresh leaves. 

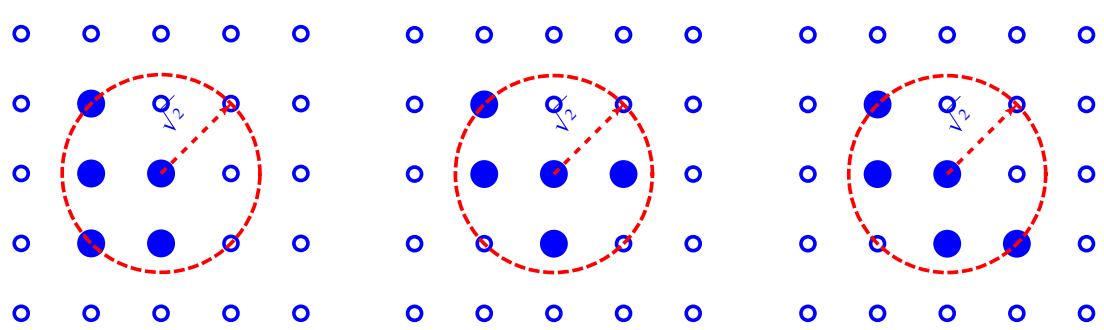

Figure 2. Versions of the clusters of five non-zero values on a regular mesh with a constant step

At the initial stage of tree construction, the probability is rather high that in all existing nodes and leaves, the value of the distribution function is zero. In this case, there is no need for a point-by-point analysis of the values of the function, and you can immediately generate a new generation for all points of the previous generation. This means a transition at each such step to a new regular uniform square mesh with a half-reduced step $h \rightarrow h / 2$. At the same time, the nodes of the old mesh are not included in the new one. There is some inefficiency in this, but, on the other hand, we more efficiently and quickly examine the behavior of a function in the parameter space.

At some point $i$, non-zero values will be detected. Each such non-zero value indicates a hit in the area, information about which will need to be detailed. But if areas of non-zero values are scattered over the momentum space and are small, some of them can be skipped on meshes with a relatively large step. It is necessary to be sure that the achieved mesh step $h_{i}$ is less than the characteristic scale of the $L_{d}$ domains in which the distribution function takes non-zero values. If there is an estimate of $L_{d}$, then the implemented procedure starts immediately with a square mesh with some step $h_{N}=2 \pi / 2^{N} \lesssim L_{d}$.

Unfortunately, at present, there are no reliable theoretical estimates of the relationship of the parameters of the simulated process with the dimensions of these domains. Criteria can be defined that allow, based on the results of calculating the distribution function on some uniform mesh, to consider that all (or almost all) regions have been found that make a significant contribution to the integral characteristics. For example, they can base on the formation of clusters of non-zero points in such areas. As the softest option, one can consider the appearance of at least one cluster of at least 5 points, the distance between which does not exceed $2 \sqrt{2} h_{i}$ (figure 2). More stringent will be the requirement that there are no single 
mesh points with non-zero values. The selection of the optimal variant of this type of criterion requires additional research.

After the uniform mesh reaches sufficient density, a transition to its adaptation by a local change in step is performed. The purpose of adaptation is to provide a fairly accurate approximation of the distribution function. As the simplest option, an adaptation procedure was implemented, based on the estimation of accuracy using a linear approximation. The procedure is implemented locally in the sense that the estimates are performed within the same branch and use points of only the last two generations.

Globality is provided at the initial iteration when the data for two complete lattices of the last generations are analyzed. Then, at each iteration with the number $i$, all the nodal points of the generation $i-1$ are considered (we conditionally identify the iteration number with the last generation number of leaves in the quadtree). The fact that these points are nodal (not leaves) means that at the previous iteration, the quality of the approximation of the distribution function for this point was unsatisfactory and its leaves were added to the mesh. For each of these points, the distribution function of each of the 4 leaves is considered. For each of these points, consider the distribution function of each of the four leaves. The reason for further splitting of each leaf is:

(1) Zero value for the sheet with a non-zero value of the parent node;

(2) A non-zero value for the sheet with zero value of the parent node;

(3) Poor approximation of the value of the opposite sheet by the non-zero values of the considered sheet and the parent node.

The mesh adaptation continues until the nodes in which the above conditions are satisfied are exhausted or until some specified minimum mesh step $h_{\min }$ is reached.

\section{Demonstration on the example of modeling the action of a single pulse}

As an example of a demonstration of the proposed approach, we consider modeling the results of the action of a pulsed electric field of the form

$$
E_{1}(t)=E_{01} \cos (2 \pi \nu t) \exp \left(-\frac{t^{2}}{2\left(\frac{\sigma}{2 \pi \nu}\right)^{2}}\right), \quad E_{2}(t)=0 .
$$

In this case, the field has a constant direction of action with which the direction of the first coordinate axis is associated. The amplitude value of the field $E_{01}$ is taken to be $300 \mathrm{~V} / \mathrm{cm}$, the frequency is $\nu=2 \mathrm{THz}$, and the 


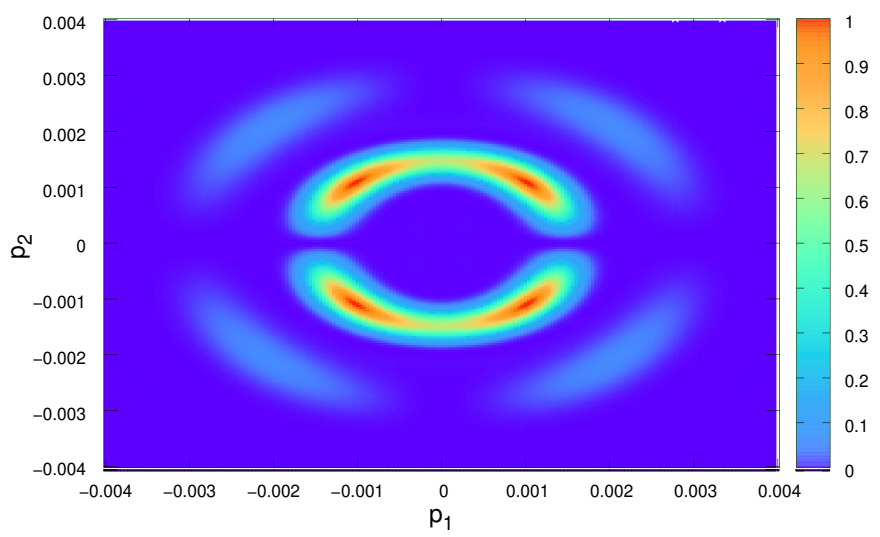

FiguRe 3. Distribution function $f\left(p_{1}, p_{2}\right)$ after the termination of the action of a field pulse with parameters: duration $2 \times 10^{-12} \mathrm{~s}$, carrier frequency $2 \times 10^{12} \mathrm{~Hz}$, amplitude $3 \times 10^{2} \mathrm{~V} / \mathrm{cm}$.

parameter $\sigma$, which determines the characteristic pulse duration of $\sigma / 2 \pi \nu$, is 6 .

The distribution function obtained by modeling with such parameters in the range $-0.00402 \leq p_{1} \leq 0.00402,-0.00402 \leq p_{2} \leq 0.00402$ on a regular square mesh with a constant step of $202 \times 202$ is shown in figure 3 . The main difference between these parameters and the case shown above in figure 1 is the field amplitude that is two orders of magnitude smaller. Nevertheless, this leads to a very radical change in the shape of the distribution function and the area of localization. On the one hand, this is an additional demonstration of the thesis about the variability of the shape of the distribution function; on the other hand, its simplification, in this case, allows us to more clearly illustrate the construction of a mesh with a variable step.

The procedure begins by considering the entire domain of definition of $f\left(p_{1}, p_{2}\right)$. A quadtree containing seven generations of nodes was built for her. For each generation, a complete uniform mesh was constructed, since at all iterations including the last seventh generation, there were only zero values in the nodes (hereinafter, non-zero values are considered $\left.f\left(p_{1}, p_{2}\right)>1.0 \times 10^{-5}\right)$. In total, the distribution function values were calculated for 21844 points. The seventh-generation mesh step was 0.049024 . From the preliminary data on the behavior of the distribution function (figure 3) it is clear that this is the expected result since the localization 

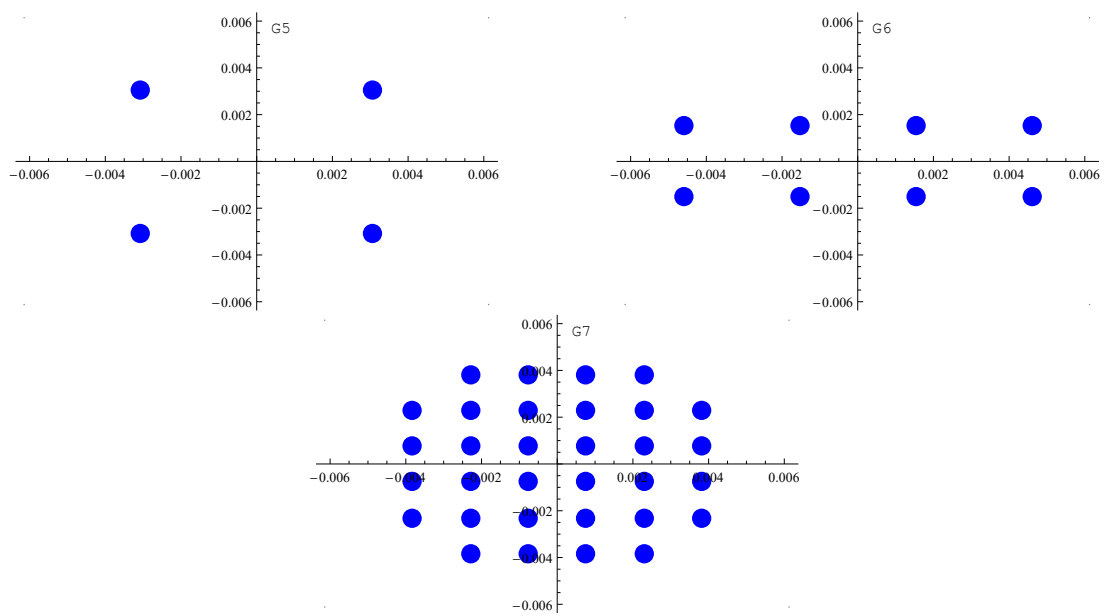

Figure 4. Non-zero values of the distribution function in generations 5, 6 and 7

region is more than an order of magnitude smaller than the achieved minimum mesh step. Therefore, it is necessary to continue squaring the mesh until non-zero values are detected.

But with the achieved size of the quadtree, the procedure for analyzing the results of each iteration and formatting the data in a sequential implementation becomes more and more time-consuming. The transition to parallel implementation of such a search is natural. Due to the independence of the search procedure for any branch of the quadtree, such parallelization can be implemented at any stage if the corresponding computing resources are available.

The parallelization is further assumed to be performed when the last current generation reaches the quadtree of size $32 \times 32=1024$. As a result, it becomes possible to search in each of the 1024 areas of size $2 \pi / 32 \times 2 \pi / 32$ independently. However, it should be borne in mind that such a search is not completely independent. The results of each iteration for all areas should be compared. If at least in one region non-zero values are found that satisfy the criteria discussed above, the global search is terminated.

At the next stage, only one region $2 \pi / 32 \times 2 \pi / 32$ in the center of the Brillouin zone was considered. For her, a new quadtree is being built, which is a continuation of part of the original. The first four non-zero values appear in it in the fifth generation. Figure 4 for the central region $-0.006128 \leq p_{1} \leq 0.006128,-0.006128 \leq p_{2} \leq 0.006128$ shows these 


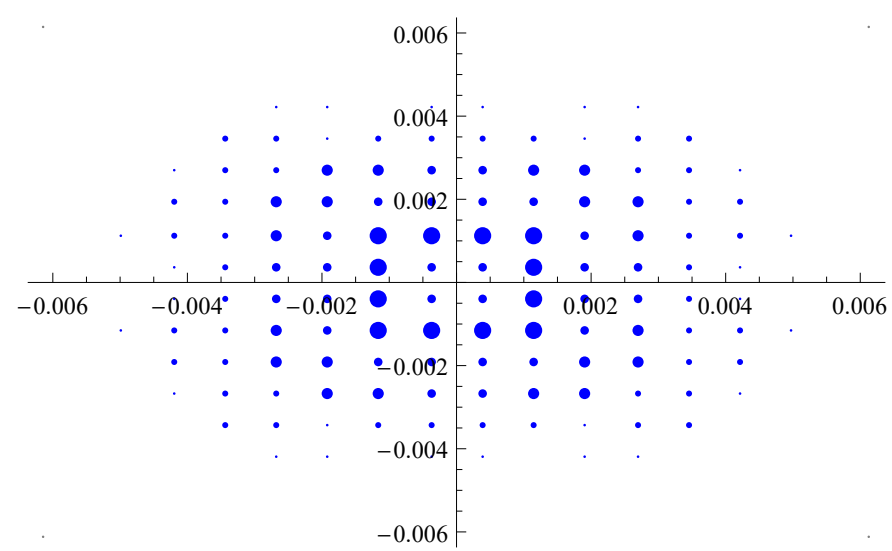

Figure 5. Non-zero values of the distribution function $\left(f\left(p_{1}, p_{2}\right)>1.0 \times 10^{-5}\right)$ on a mesh with cell size $0.000766 \times$ 0.000766 . The sizes of the points are proportional to the value of the distribution function.

points and points with non-zero values of the sixth and seventh generations (there are 8 and 20 respectively).

The solid-mesh work is completed on the next generation with a covering square of $0.000766 \times 0.000766$. The result of calculating the distribution function values on this mesh is shown in figure 5 . The number of not zero points is 132 , and the maximum value of the distribution function reaches 0.7425. In contrast to the previous one, in this figure, the points have five sizes in accordance with the function falling into the intervals of values with the boundaries $1.0,10^{-1}, 10^{-2}, 10^{-3}, 10^{-4}, 10^{-5}$.

At this stage, it was assumed that the distribution function is localized in the region shown in figures 4 and 5 . In total, values for 341 points were determined in this area (of which 176 are not zero).

Further adaptation of the mesh was carried out in five stages, i.e., five more generations of nodes were built according to the procedure described above, but now they were completed only in the areas of transition to zero values and high gradients of the distribution function. Figures 6 and 7 show the results of the first $(+288$ points) and second $(+384$ points $)$ stages (only points with non-zero values). The sizes of the points are also ranked in accordance with the values of the distribution function in them, but with a smaller scale. At the next three stages, 272, 176 and 176 points were completed and counted, respectively. At the last stage, the step of the completed mesh became smaller than the step of a homogeneous mesh, the results of which are shown in figure 3 .

The target parameter for evaluating the quality of the mesh was 


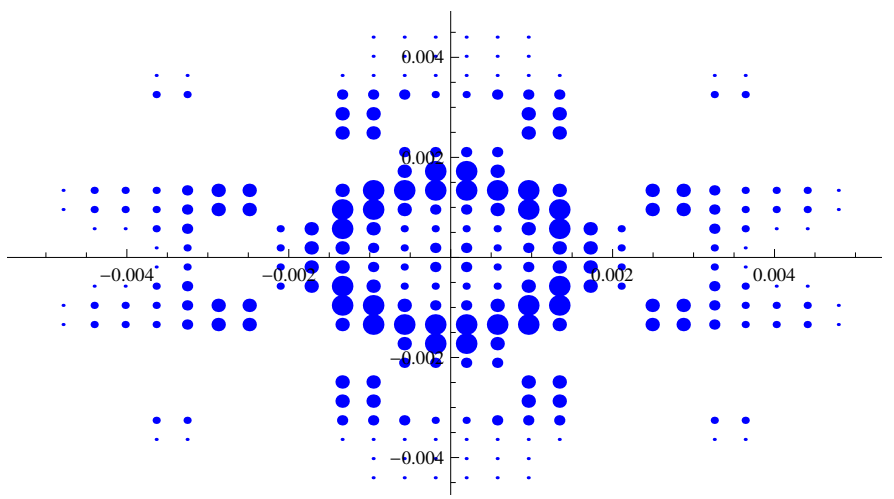

Figure 6. The first stage of mesh adaptation

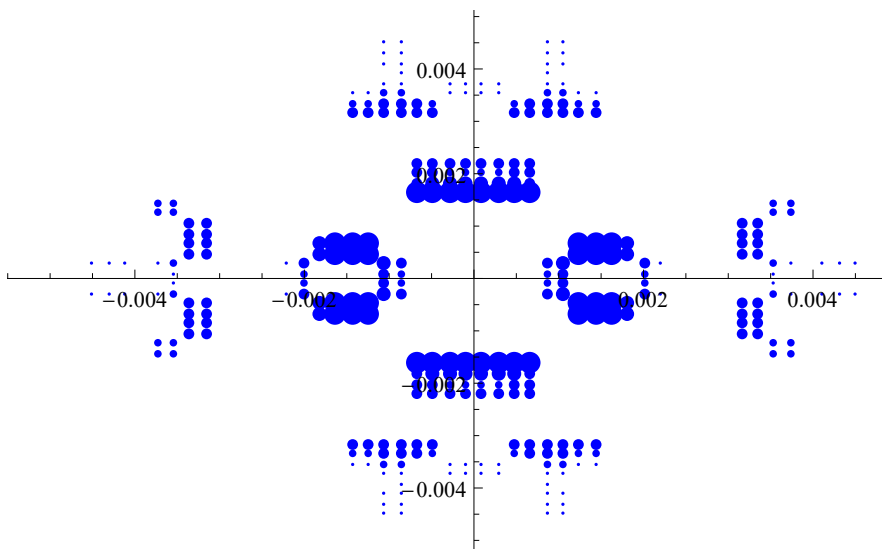

Figure 7 . The second stage of mesh adaptation

the carrier density calculated at each stage. The reference value was $1.01278 \times 10^{9} 1 / \mathrm{cm}^{2}$, obtained on a homogeneous mesh. The results presented in Table 1 show that the target integral value converges quickly and stably. Already on meshes of the order of a thousand nodes, the test value obtained on a uniform mesh of 40804 nodes is well reproduced. It seems interesting to study in detail the behavior of integral characteristics calculated on adaptive meshes of the type presented.

\section{Conclusion}

A similar problem appears in modeling the processes of production of electron-positron pairs in quantum electrodynamics (QED), [10-12]. In the general statement in the case of $\mathrm{QED}$, the problem is three-dimensional. In 
TABLE 1. The results of calculating the density of carriers on different meshs

\begin{tabular}{lc}
\hline \multicolumn{1}{c}{ Used mesh } & $\begin{array}{c}\text { Carrier density } \\
1 / \mathrm{cm}^{2}\end{array}$ \\
\hline Uniform mesh $202 \times 202=40804$ nodes & $1.012780 \times 10^{9}$ \\
Quadro tree in the area of localization 341 nodes & $0.967066 \times 10^{9}$ \\
First step of adaptation $341+288=629$ nodes & $1.002584 \times 10^{9}$ \\
Second step of adaptation $629+384=1013$ nodes & $1.013377 \times 10^{9}$ \\
Third step of adaptation $1013+272=1285$ nodes & $1.014233 \times 10^{9}$ \\
Fourth step of adaptation $1285+176=1461$ nodes & $1.014268 \times 10^{9}$ \\
Fifth step of adaptation $1461+176=1637$ nodes & $1.014269 \times 10^{9}$ \\
\hline
\end{tabular}

this case, the construction of an adaptive mesh is possible by a similar algorithm in the octree format. But there is a large class of problems in which, due to various types of symmetries of the external field, it is possible to limit oneself to two dimensions, for example, $[13,14]$. In these cases, the solution proposed in this paper is applicable with minimal modifications.

The presented results make it possible to accelerate and automate the procedure for constructing computational meshes for numerical simulation of carrier generation processes in two-dimensional media (or for two-dimensional problems in three-dimensional media). The proposed approach has great potential for developing the procedures used and improving methods for evaluating the results obtained. The paper contains results of testing and evaluation on the example of the problem of modeling the reaction of graphene to the action of an external electric field with realistic parameters.

\section{References}

[1] M.M. Glazov, S.D. Ganichev. "High frequency electric field induced nonlinear effects in graphene", Physics Reports, 535:3 (2014), pp. 101-138. do) $\uparrow_{93}$

[2] P. Bowlan, E. Martinez-Moreno, K. Reimann, T. Elsaesser, M. Woerner. "Ultrafast terahertz response of multilayer graphene in the nonperturbative regime", Phys. Rev. B, 89:4 (2014), 041408. dot $\uparrow_{93,95}$

[3] M. Baudisch, A. Marini, J.D. Cox, T. Zhu, F. Silva, S. Teichmann, M. Massicotte, F. Koppens, L.S. Levitov, F.J. Garcia de Abajo, J. Biegert. "Ultrafast nonlinear optical response of Dirac fermions in graphene", Nature Communications, 9 (2018), 1018. do $\uparrow_{93}$

[4] Zi-Yu Chen, Rui Gin. "Circularly polarized extreme ultraviolet high harmonic generation in graphene", Optics Express, 27:3 (2019), pp. 3761-3770. do $\uparrow_{93}$

[5] S.A. Smolyansky, D.V. Churochkin, V.V. Dmitriev, A.D. Panferov, B. Kämpfer. "Residual currents generated from vacuum by an electric field 
pulse in 2+1 dimensional QED models", EPJ Web of Conferences, 138 (2017), 06004. dot $\uparrow_{94}$

[6] A.D. Panferov, S.A. Smolyansky, D.B. Blaschke, N.T. Gevorgyan. "Comparing two different descriptions of the I-V characteristic of graphene: theory and experiment", EPJ Web of Conferences, 204 (2019), 06008. dot $\uparrow_{94}$

[7] S.A. Smolyansky, A.D. Panferov, D.B. Blaschke, N.T. Gevorgyan. "Nonperturbative kinetic description of electron-hole excitations in graphene in a time dependent electric field of arbitrary polarization", Particles, 2:2 (2019), pp. 208-230. do $\uparrow_{94,95}$

[8] A.D. Panferov, A.V. Makhankov. "Simulation of the effect of short optical pulses on graphene", Program Systems: Theory and Applications, 10:1(40) (2019), pp. 47-58.

[9] A.A. Trunov, R.D. Al'-Karavi, T.T. Verevin, N.A. Novikov, A.D. Panferov. "Visualization and analysis of data arrays in modeling the behavior of graphene in an external electric field", Informatsionnyye tekhnologii $i$ matematicheskoye modelirovaniye, Materialy XVIII Mezhdunarodnoy konferentsii imeni A. F. Terpugova. V. 1, ITMM-2019 (26-30 iyunya 2019 g., Saratov, Rossiya), Izdatel'stvo NTL, Tomsk, 2019, ISBN 978-5-89503-628-0, pp. 158-162 (in Russian). URL $* \uparrow_{95}$

[10] J.S. Schwinger. "On gauge invariance and vacuum polarization", Phys. Rev., 82:5 (1951), pp. 664-679. dot $\uparrow_{102}$

[11] V.N. Pervushin, V.V. Skokov. "Kinetic description of fermion production in the oscillator representation", Acta Phys. Polon. B, 37 (2006), pp. 25872600. URL) $\uparrow_{102}$

[12] F. Hebenstreit, R. Alkofer, H. Gies. "Pair production beyond the Schwinger formula in time-dependent electric fields", Phys. Rev. D, 78:6 (2008), 061701.

[13] D.B. Blaschke, B. Kämpfer, S.M. Schmidt, A.D. Panferov, A.V. Prozorkevich, S.A. Smolyansky. "Properties of the electron-positron plasma created from a vacuum in a strong laser field: Quasiparticle excitations", Phys. Rev. D, 88:4 (2013), 045017. do $\uparrow_{103}$

[14] A.D. Panferov, S.A. Smolyansky, A. Otto, B. Kämpfer, D.B. Blaschke, L. Juchnowski. "Assisted dynamical Schwinger effect: pair production in a pulsed bifrequent field", Eur. Phys. J. D, 70 (2016), 56. do) $\uparrow_{103}$

$\begin{array}{ll}\text { Received } & 05.02 .2020 \\ \text { Revised } & 20.03 .2020 \\ \text { Published } & 26.03 .2020\end{array}$

Recommended by prof. S. M. Abramov 
Sample citation of this publication:

Anatolii D. Panferov, Alexey V. Makhankov, Alexander V. Trunov. "The use of an adaptive mesh based on a quadtree for modeling the final state of a quantum field system under pulsed external action". Program Systems: Theory and Applications, 2020, 11:1(44), pp. 93-105.

do) $10.25209 / 2079-3316-2020-11-1-93-105$

(URL) http://psta.psiras.ru/read/psta2020_1_93-105.pdf

The same article in Russian:

10.25209/2079-3316-2020-11-1-79-92

About the authors:
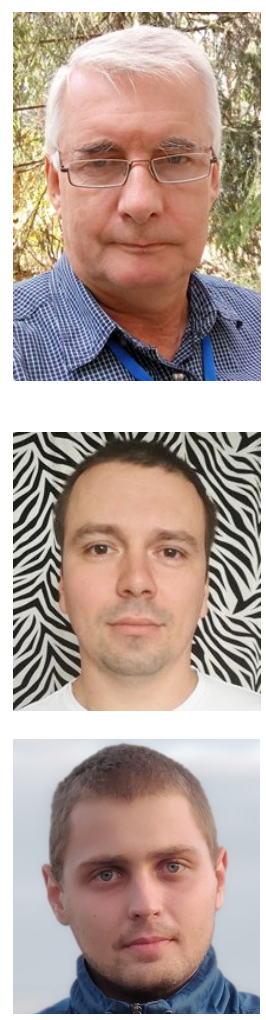

\section{Anatolii Dmitrievich Panferov}

$\mathrm{PhD}$ in Physics and Mathematics, Deputy Head PRCNIT SSU on research and production activities. Associate Professor of the Department Discrete Mathematics and Information Technology SSU. Research interests: high-performance computing, parallel programming, numerical solution of quantum kinetic equations, modeling of the vacuum production of particles in QED, generation of carriers in semiconductors including gapless, processes in the early stages of collision of relativistic nuclei.

$$
\begin{array}{ll}
\text { (D) } & \text { 0000-0003-2332-0982 } \\
\text { e-mail: } & \text { panferovad@info.sgu.ru }
\end{array}
$$

\section{Alexey Vladimirovich Makhankov}

Graduate student of the SSU. Research interests: modeling of physical processes on high-performance computing systems, parallel programming.

$$
\begin{array}{ll}
\text { e-mail: } & \text { neo0-0002-9848-9734 } \\
\text { next642009@yandex.ru }
\end{array}
$$

\section{Alexander Vladimirovich Trunov}

Graduate student of the SSU. Research interests: modeling of physical processes on high-performance computing systems, the use of parallel programming for solving applied problems, modeling of virtual devices in the LabVIEW graphical programming environment.

$$
\begin{array}{ll}
\text { (iD) } & \text { 0000-0003-2831-6763 } \\
\text { e-mail: } & \text { snek271@yandex.ru }
\end{array}
$$

\footnotetext{
Эта же статья по-русски: dot 10.25209/2079-3316-2020-11-1-79-92
} 